This is the peer reviewed version of the following article: Fisher, C. Y., Adams, J., Frawley, J. E., Hickman, L. D. and Sibbritt, D. W. (2018), Is there a role for Western herbal medicine in treating cyclic perimenstrual pain and discomfort?. Aust N Z J Obstet Gynaecol., which has been published in final form at https://doi.org/10.1111/ ajo.12883. This article may be used for non-commercial purposes in accordance with Wiley Terms and Conditions for Self-Archiving. 
Is there a role for Western herbal medicine in treating cyclic perimenstrual pain and discomfort?

\author{
Ms Carole Y FISHER BSc ${ }^{1}$, Jon ADAMS PhD ${ }^{1}$, Jane E FRAWLEY PhD ${ }^{1}$, Louise D \\ HICKMAN PhD ${ }^{1}$, David W SIBBRITT PhD ${ }^{1}$ \\ ${ }^{1}$ Faculty of Health, University of Technology Sydney, New South Wales, Australia
}

\title{
Keywords
}

Cyclic perimenstrual pain and discomfort, dysmenorrhea, Herbal medicine, irregular periods, premenstrual syndrome, Vitex agnus-castus

\begin{abstract}
Conventional treatments for cyclic perimenstrual pain and discomfort whilst numerous and diverse, have drawbacks including side effects, interference with women's reproductive function and, importantly, failure to address symptoms. Many women turn to herbal medicine to treat the myriad menstrual symptoms. Clinical evidence supports the efficacy of Vitex agnus-castus but other medicinal herbs typically used by Western herbalists for treating menstrual symptoms are unsupported by clinical trials. This raises concerns around the efficacy and safety of these herbs. Women's treatment options need to be extended and individualised, where current conventional strategies fail, requiring appropriate clinical trials of potentially useful herbal medicines.
\end{abstract}




\section{Is there a role for Western herbal medicine in treating cyclic perimenstrual pain and discomfort?}

Conventional treatments for cyclic perimenstrual pain and discomfort (CPPD) are diverse and numerous ${ }^{1}$. Many of these treatments have drawbacks including significant costs to users and the health system, some unacceptable side effects ${ }^{1}$, interference with women's reproductive function and, importantly, for up to $40 \%$ of sufferers a failure to address their symptoms ${ }^{1}$. In response, many women have opted to use complementary and alternative medicine (CAM) - a range of diverse health-related practices and products located predominantly outside the conventional medical curriculum and profession ${ }^{2}$ - to treat CPPD symptoms ${ }^{2-4}$. In particular, women who experienced endometriosis and/or frequent premenstrual syndrome (PMS), irregular periods or dysmenorrhea were significantly more likely to consult a naturopath/herbalist than women who did not suffer from these menstrual issues $^{2}$.

Reliable, robust, scientific evidence regarding the efficacy and safety of different CAM for treating CPPD is required to convince stakeholders, including conventional medical practitioners, that such treatments are viable, effective and safe. Such evidence is also important to health policymakers' efforts to fund the efficient integration of proven CAM thereby extending CPPD treatment options available to women.

While many Western herbalists (WHs) prescribe medicinal herb combinations tailored to women's needs, Vitex agnus-castus (chaste tree), Paeonia lactiflora (white peony), Angelica sinensis (Dong quai) and Actaea racemosa (black cohosh) are core herbs favoured for treating all four CPPD symptoms. WHs perceive there to be a high level of efficacy for herbal 
medicine in treating dysmenorrhea, PMS, heavy and irregular periods, with low levels of adverse effects and a potentially positive cost to benefit ratio ${ }^{5}$.

So the questions addressed here are two-fold - is herbal medicine a viable CPPD treatment option? And how well supported are the efficacy and safety of different medicinal herbs in treating CPPD?

\section{Vitex agnus-castus (VAC)}

VAC is one of the most investigated medicinal herbs for the treatment of CPPD symptoms. Since 2001, seven published systematic reviews have reported that : VAC reduced premenstrual dysphoric disorder (PMDD) symptoms by more than $50 \%$ after 2 months, an effect equal to selective serotonin reuptake inhibitors ${ }^{6}$; VAC was equal to or better than vitamin B6 in treating PMS, with both providing around $48 \%$ improvement ${ }^{6}$; and efficacy rates for VAC were between $52 \%$ to $85 \%$ compared to those for placebo which were between $24 \%$ to $56 \%$, after 3 cycles of treatment ${ }^{6}$ with improvement in both physical and psychological domains in PMS and PMDD ${ }^{6}$. Use of oral contraceptive pills did not appear to affect the results achieved with $\mathrm{VAC}^{6}$ and clinical trials of VAC treatment in dysmenorrhea, have found it to be as effective as oral contraceptives ${ }^{7}$ and mefenamic acid in menstrual pain reduction ${ }^{8}$. In treating cyclical mastalgia VAC reduced breast discomfort by between $45 \%$ to $85 \%$ within three months of treatment ${ }^{9}$.

According to a systematic review of the adverse events (AEs) associated with VAC, these were assessed as mild, reversible and equivalent to placebo. AEs with the highest incidence, reported by $2-5 \%$ of 9335 users, were nausea, gastro-intestinal disturbances, headache and 
acne ${ }^{10}$. Apart from a single serious AE of peripheral ischemia, deemed as a 'probable' result of VAC, its use is considered to be without serious risks ${ }^{6,10}$. There is a theoretical possibility that VAC could interact with drugs that modulate dopamine and pituitary-based hormone therapies although no such interactions have yet been reported ${ }^{10}$. Although VAC was found to have no effect on the composition of breast milk its safety during pregnancy and lactation has not been established ${ }^{10}$.

\section{Angelica sinensis, Paeonia lactiflora and Actaea racemosa}

For Angelica sinensis, Paeonia lactiflora and Actaea racemosa, there are no available clinical trials corroborating their efficacy in treating CPPD. Scientific evidence of their potential benefits for CPPD relies on pre-clinical studies of pharmacological activity in what is a complicated, incompletely understood and multifactorial etiology ${ }^{1}$.

What we do know from pre-clinical data for Angelica, that may be relevant to CPPD treatment, includes a potential: for anti-inflammatory activity ${ }^{11,}$; for reducing stress/depression via a brain neurotrophic factor ${ }^{12}$; for normalising blood cell profiles ${ }^{11}$; as a progesterone antagonist reducing heavy bleeding ${ }^{13}$ and to alter mood and relax muscles through GABAminergic ${ }^{14}$ and serotonergic receptor sites ${ }^{15}$. Putative phyto-estrogenic activity may also mimic reproductive hormone therapy ${ }^{13}$. However, Angelica's anticoagulant properties contra-indicates its use in heavy periods ${ }^{11}$. As for Paeonia, it has been shown to have potential anti-depressant activity, raising serotonin and dopamine levels, through inhibition of monoamine oxidase activity ${ }^{16}$ and it may also relieve dysmenorrhea through 
analgesic ${ }^{17}$ and anti-inflammatory ${ }^{17}$ properties, inhibiting both leukotriene B4 and prostaglandin E2 production ${ }^{17}$.

Although traditionally used in treating menstrual dysfunction ${ }^{18}$ no trials have assessed Actaea's effectiveness in alleviating menstrual problems. Actaea lacks hormonal activity ${ }^{18}$, but pre-clinical studies indicate it is anti-inflammatory ${ }^{19}$, analgesic ${ }^{19}$ and has GABA-A ${ }^{20}$ binding properties, possibly therefore relieving dysmenorrhea. Actaea also interacts at serotenergic ${ }^{18}$, dopaminergic ${ }^{18}$ and opioid ${ }^{18}$ receptor sites potentially influencing moodrelated PMS issues. Additionally, Actaea may alter reproductive hormone levels through pituitary function ${ }^{18}$ and reduce excessive bleeding by inhibiting plasminogen activation ${ }^{21}$. So whilst preclinical data indicate Actaea has the potential to benefit CPPD, credible evidence of its clinical efficacy is lacking.

\section{So is Western herbal medicine an option for CPPD?}

Currently, evidence suggests a minimum efficacy of $50 \%$ and a good safety profile for VAC as a treatment option for CPPD, particularly PMS/PMDD. However, the other commonly used medicinal herbs at present have little scientific validation of their efficacy via clinical trials although none of them have been associated with serious AE concerns, when properly sourced ${ }^{22}$.

There remains little objective, scientific support for three of the four core medicinal herbs used by WHs for CPPD patients. This does not necessarily preclude them from having a valuable place in the treatment of CPPD. Lack of financial incentives hamper more extensive 
research of medicinal herbs through RCTs that could verify their efficacy in CPPD treatment, but in an environment demanding greater scientific corroboration of CAM, anecdotal evidence and traditional credentials alone are insufficient to encourage its acceptance. Conventional health providers and policymakers will inevitably remain sceptical and wary of adopting Western herbal medicine without more compelling scientific evidence.

Seeking out additional treatments are none-the-less desirable given the current failure of conventional therapies to effectively treat CPPD. Currently, conventional health practitioners and academics in the field of gynecology have urged the need for treatments to be individualised, possibly requiring concurrent use of several treatment approaches ${ }^{5}$. Inherent in WHs' practice is the blending of medicinal herbs, tailored to address the individual woman's symptoms, much in line with the recommendation for individualising treatment. While credible, high quality, clinical studies of medicinal herbs and the practice of WHs are needed, a realistic paradigm by which to assess their efficacy in treating women with CPPD is also required, one that fulfills the broader need for using individualised therapy in CPPD patient care.

Women are using Western herbal medicines and consulting with WHs to treat CPPD, irrespective of the paucity of robust clinical trials to support much of this practice. This raises concerns around efficacy and safety that need to be addressed with rigorous scientific investigation. Meanwhile, there is a need for additional options to improve the current treatment of CPPD, for which WHs and herbal medicines, may offer real benefits to women, policymakers and practitioners, none more so than conventional health providers who require such information and assurance in order to effectively and safely coordinate care for women with CPPD. 


\section{References}

1. Marjoribanks J, Ayeleke RO, Farquhar C, Proctor M. Nonsteroidal anti-inflammatory drugs for dysmenorrhoea. The Cochrane database of systematic reviews. 2015;7:CD001751.

2. Fisher C, Adams J, Hickman L, Sibbritt D. The use of complementary and alternative medicine by 7427 Australian women with cyclic perimenstrual pain and discomfort: a cross-sectional study. BMC Complementary and Alternative Medicine. 2016;16(1):1-11.

3. Chao MT, Abercrombie PD, Nakagawa S, Gregorich SE, Learman LA, Kuppermann M. Prevalence and Use of Complementary Health Approaches among Women with Chronic Pelvic Pain in a Prospective Cohort Study. Pain Medicine (United States). 2015;16(2):328-340.

4. Smith CA, Bateson DJ, Weisberg E. A survey describing the use of complementary therapies and medicines by women attending a family planning clinic. BMC Complementary and Alternative Medicine. 2013;13.

5. Fisher C, Adams J, Frawley J, Hickman L, Sibbritt D. Western herbal medicine consultations for common menstrual problems; practitioner experiences and perceptions of treatment. Phytotherapy Research. 2018;32(3):531-541.

6. van Die MD, Burger HG, Teede HJ, Bone KM. Vitex agnus-castus Extracts for Female Reproductive Disorders: A Systematic Review of Clinical Trials. Planta Med. 13.05.2013 2013;79(07):562-575.

7. Aksoy AN, Gozukara I, Kucur SK. Evaluation of the efficacy of Fructus agni casti in women with severe primary dysmenorrhea: A prospective comparative Doppler study. Journal of Obstetrics and Gynaecology Research. 2014;40(3):779-784.

8. Zeraati F, Shobeiri F, Nazari M, Araghchian M, Bekhradi R. Comparative evaluation of the efficacy of herbal drugs (fennelin and vitagnus) and mefenamic acid in the treatment of primary dysmenorrhea. Iranian Journal of Nursing \& Midwifery Research. 2014;19(6):581584.

9. Carmichael AR. Can Vitex Agnus castus be used for the treatment of mastalgia? What is the current evidence? Evidence-based Complementary and Alternative Medicine. 2008;5(3):247250.

10. Daniele C, Coon JT, Pittler MH, Ernst E. Vitex agnus castus: A systematic review of adverse events. Drug Safety. 2005;28(4):319-332.

11. Li PL, Sun HG, Hua YL, et al. Metabolomics study of hematopoietic function of Angelica sinensis on blood deficiency mice model. Journal of Ethnopharmacology. 2015;166:261-269.

12. Shen J, Zhang J, Deng M, Liu Y, Hu Y, Zhang L. The Antidepressant Effect of Angelica sinensis Extracts on Chronic Unpredictable Mild Stress-Induced Depression Is Mediated via the Upregulation of the BDNF Signaling Pathway in Rats. Evidence-based Complementary and Alternative Medicine. 2016;2016.

13. Ahmed HM, Yeh JY, Tang YC, Cheng WTK, Ou BR. Molecular screening of Chinese medicinal plants for progestogenic and anti-progestogenic activity. Journal of Biosciences. 2014;39(3):453-461.

14. Deng $S$, Chen SN, Lu J, et al. GABAergic phthalide dimers from Angelica sinensis (Oliv.) diels. Phytochemical Analysis. 2006;17(6):398-405.

15. Deng $S, C h e n S N$, Yao $P$, et al. Serotonergic activity-guided phytochemical investigation of the roots of Angelica sinensis. Journal of Natural Products. 2006;69(4):536-541.

16. Yu XH, Song T, Hou XL, et al. Anti-depressant effect of paeonia lactiflora pall extract in rats. Tropical Journal of Pharmaceutical Research. 2017;16(3):577-580.

17. He D-Y, Dai S-M. Anti-Inflammatory and Immunomodulatory Effects of Paeonia Lactiflora Pall., a Traditional Chinese Herbal Medicine. Frontiers in Pharmacology. 2011-February-25 2011;2(10). 
18. Mercado-Feliciano M, Cora MC, Witt KL, et al. An ethanolic extract of black cohosh causes hematological changes but not estrogenic effects in female rodents. Toxicology and Applied Pharmacology. 2012;263(2):138-147.

19. Kim SJ, Kim MS. Inhibitory effects of cimicifugae rhizoma extracts on histamine, bradykinin and COX-2 mediated inflammatory actions. Phytotherapy Research. 2000;14(8):596-600.

20. Cicek SS, Khom S, Taferner B, Hering S, Stuppner H. Bioactivity-guided isolation of GABAa receptor modulating constituents from the rhizomes of Actaea racemosa. Journal of Natural Products. 2010;73(12):2024-2028.

21. Lee DY, Roh CR, Kang YH, et al. Effects of black cohosh on the plasminogen activator system in vascular smooth muscle cells. Maturitas. 2013;76(1):75-80.

22. Izzo AA, Hoon-Kim S, Radhakrishnan R, Williamson EM. A Critical Approach to Evaluating Clinical Efficacy, Adverse Events and Drug Interactions of Herbal Remedies. Phytotherapy Research. 2016;30(5):691-700. 